\title{
An approximation result for solutions of Hessian equations
}

\section{John Urbas}

Accepted: 28 June 2006 / Published online: 21 October 2006

(C) Springer-Verlag 2006

\begin{abstract}
We show that $W^{2, p}$ weak solutions of the $k$-Hessian equation $F_{k}\left(D^{2} u\right)=$ $g(x)$ with $k \geq 2$ can be approximated by smooth $k$-convex solutions $v_{j}$ of similar equations with the right hands sides controlled uniformly in $C^{0,1}$ norm, and so that the quantities $\int_{B_{r}}\left(\Delta v_{j}\right)^{p-k+1} F_{k-1}\left(D^{2} v_{j}\right)$ are bounded independently of $j$. This result simplifies the proof of previous interior regularity results for solutions of such equations. It also permits us to extend certain estimates for smooth solutions of degenerate two dimensional Monge-Ampère equations to $W^{2, p}$ solutions.
\end{abstract}

Mathematics Subject Classification (2000) Primary 35J60; Secondary

\section{Introduction}

In recent work $[14,15]$ we showed that viscosity solutions $u \in W^{2, p}(\Omega)$ of the $k$-Hessian equation

$$
F_{k}\left(D^{2} u\right)=g(x) \quad \text { in } \Omega \subset \mathbf{R}^{n}
$$

belong to $C^{1,1}(\Omega)$ if $g \in C^{1,1}(\Omega)$ is positive and $p>k(n-1) / 2$. $C^{2, \alpha}(\Omega)$ regularity then follows from the Evans-Krylov estimates $[4,6]$ and higher regularity follows from standard linear elliptic theory [5] in accordance with the smoothness of $g$. Here

$$
F_{k}\left(D^{2} u\right)=\sigma_{k}\left(\lambda_{1}, \ldots, \lambda_{n}\right)=\sum_{1 \leq i_{1}<\cdots<i_{k} \leq n} \lambda_{i_{1}} \cdots \lambda_{i_{k}}
$$

Supported by an Australian Research Council Senior Fellowship.

J. Urbas $(\varangle)$

Centre for Mathematics and its Applications, Mathematical Sciences Institute,

Australian National University, Canberra, ACT 0200, Australia

e-mail: urbas@maths.anu.edu.au 
where $\lambda_{1}, \ldots, \lambda_{n}$ are the eigenvalues of $D^{2} u$. It is well known that (1.1) is an elliptic equation on solutions such that $\lambda=\left(\lambda_{1}, \ldots, \lambda_{n}\right)$ belongs to the cone

$$
\Gamma_{k}=\left\{\lambda \in \mathbf{R}^{n}: \sigma_{j}(\lambda)>0 \text { for } j=1, \ldots, k\right\} .
$$

Furthermore, $F_{k}\left(D^{2} u\right)^{1 / k}$ is a concave function of $D^{2} u$ for such solutions [1]. We refer to such solutions as $k$-convex.

The estimates in $[14,15]$ can be proved much more simply for smooth solutions. What complicates the proofs considerably is the approximation argument required to prove these estimates for $W^{2, p}$ solutions. In [14,15] we used a technical argument involving regularization and second order difference quotients to achieve this. The whole procedure would be simplified if it were possible to approximate a $W^{2, p}$ viscosity solution $u$ of (1.1) weakly in $W_{\text {loc }}^{2, p}$ by smooth solutions $\left\{u_{j}\right\}$ of similar equations $F_{k}\left(D^{2} u_{j}\right)=g_{j}(x)$ in such a way that the right hand sides $g_{j}$ are controlled uniformly in a suitable norm, for example, in $C^{1,1}$ or in $C^{0,1}$, and are uniformly bounded away from zero.

This is of course trivial for the linear case $k=1$ : the usual regularization $u_{\epsilon}$ belongs to $C^{\infty}(\Omega)$ and converges to $u$ in $W_{\text {loc }}^{2, p}(\Omega)$ for $1 \leq p<\infty$. But this is no longer clear in the nonlinear case. If $k \geq 2$, all we can say a priori is that

$$
g_{\epsilon}:=F_{k}\left(D^{2} u_{\epsilon}\right) \rightarrow F_{k}\left(D^{2} u\right)=g \quad \text { in } L_{\mathrm{loc}}^{p / k}(\Omega)
$$

if $u \in W^{2, p}(\Omega)$, which is much too weak for our purposes. To apply the estimates in $[14,15]$ to $u_{\epsilon}$ we need to know at least that $g_{\epsilon}$ is uniformly bounded in $C^{1}(\Omega)$ and bounded away from zero.

Our main result here states that it is possible to approximate $u$ locally by smooth solutions of similar equations in such a way that the key quantities that need to be controlled in the proofs in $[14,15]$ remain uniformly controlled, and such that the right hand sides of the equations remain uniformly bounded in $C^{1}(\Omega)$ and bounded away from zero if $g$ is positive. More precisely, we will prove the following.

Theorem 1.1 Let $u \in W^{2, p}(\Omega)$ be a viscosity solution of (1.1) in a domain $\Omega \subset \mathbf{R}^{n}$, where $g \in C^{0,1}(\Omega)$ is positive and $p \geq k \geq 2$. Then for any point $x_{0} \in \Omega$ there exist a number $r>0$ and smooth $k$-convex solutions $\left\{v_{j}\right\}$ of equations $F_{k}\left(D^{2} v_{j}\right)=g_{j}(x)$ in $B_{r}=B_{r}\left(x_{0}\right)$ such that

(i) $\quad v_{j} \rightarrow u$ uniformly in $B_{r}$;

(ii) $\left\|v_{j}\right\|_{C^{1}\left(\bar{B}_{r}\right)}$ is bounded independently of $j$;

(iii) $\int_{B_{r}}\left(\Delta v_{j}\right)^{p-k+1} F_{k-1}\left(D^{2} v_{j}\right)$ is bounded independently of $j$;

(iv) $\left\|g_{j}\right\|_{C^{1}\left(\bar{B}_{r}\right)} \leq 1+\|g\|_{C^{0,1}\left(\bar{B}_{2 r}\right)}$ and $\inf _{B_{r}} g_{j} \geq \inf _{B_{r}} g$ for all $j$.

The proof of the interior second derivative bound in [15] involves an iteration of integrals of the kind appearing in (iii), and the exponent of integrability increases provided $p>k(n-1) / 2$ (for this we need $g$ to be positive). It then follows from the results of [15] applied to $v_{j}$ that $\left\|v_{j}\right\|_{C^{1,1}\left(\bar{B}_{r / 2}\right)}$ is bounded independently of $j$, and the same is true of $\left\|v_{j}\right\|_{C^{2, \alpha}\left(\bar{B}_{r / 4}\right)}$ by the Evans-Krylov estimates. Thus by (i), $u$ satisfies similar bounds. Therefore we have the following.

Theorem 1.2 Let $u \in W^{2, p}(\Omega)$ be a viscosity solution of (1.1) in a bounded domain $\Omega$ in $\mathbf{R}^{n}$, where $g \in C^{0,1}(\Omega)$ is positive and $p>k(n-1) / 2$. Then $u$ belongs to $C^{2, \alpha}(\Omega)$ for any $\alpha \in(0,1)$. 
This is a slight improvement of the corresponding regularity result in [15] in that here we assume $g \in C^{0,1}(\Omega)$ rather than $g \in C^{1,1}(\Omega)$.

A more interesting improvement can be obtained by applying an estimate of Liu and Bao [7] to the approximations $v_{j}$. We see then that the conclusion of Theorem 1.2 is valid for $p>k(k-1)$, provided also that $g \in C^{1,1}(\Omega)$ is positive (this gives a better lower bound on the integrability exponent $p$ if $k<n / 2)$. The optimal value of $p$ is an interesting open problem. Examples in $[9,13]$ show that $p \geq k(k-1) / 2$ is necessary if $k \geq 3$.

We can also use a minor modification of Theorem 1.1 to extend the $C^{1, \alpha}$ estimates proved in [16] for smooth solutions of degenerate two dimensional Monge-Ampère equations to $W^{2, p}$ solutions.

Theorem 1.3 Let $u \in W^{2, p}(\Omega), p \geq 2$, be a convex generalized solution of

$$
\left(\operatorname{det} D^{2} u\right)^{1 / 2}=\tilde{g}(x) \quad \text { in } \Omega \subset \mathbf{R}^{2}
$$

where $\tilde{g} \in W^{1, p}(\Omega)$ is nonnegative. Then $u \in C^{1, \alpha}(\Omega)$ for $\alpha=1-1 / p$, and for any concentric balls $B_{R} \subset B_{2 R} \subset \subset \Omega$ we have

$$
[D u]_{\alpha ; B_{R}} \leq C R^{-1 / p}\left\{\left(\int_{B_{2 R}}(\Delta u)^{p}\right)^{1 / p}+\left(\int_{B_{2 R}} \tilde{g}^{p}\right)^{1 / p}+\left(R^{p} \int_{B_{2 R}}|D \tilde{g}|^{p}\right)^{1 / p}\right\}
$$

where $C$ depends only on $p$, and $[D u]_{\alpha ; B_{R}}$ denotes the Hölder seminorm of Du given by

$$
[D u]_{\alpha ; B_{R}}=\sup _{\substack{x, y \in B_{R} \\ x \neq y}} \frac{|D u(x)-D u(y)|}{|x-y|^{\alpha}} .
$$

In [16] we also proved a variant of Theorem 1.3 for smooth solutions in the case $1<p<2$. We have not been able to extend this result to $W^{2, p}$ solutions.

From [3, Lemma 1] it follows that

$$
F_{k-1}\left(D^{2} u\right) \geq c(k, n) g^{\frac{k-2}{k-1}}(\Delta u)^{\frac{1}{k-1}},
$$

where $c(k, n)$ is a positive constant. Consequently, if $g$ is bounded away from zero, it follows from Theorem 1.1 (iii) and the $L^{p}$ theory [5, Chap. 9] that the $W^{2, p-k+1+1 /(k-1)}$ $\left(B_{r / 2}\right)$ norms of $v_{j}$ are uniformly bounded. Therefore, by passing to a subsequence if necessary, we may assume that $v_{j}$ converges weakly in $W^{2, p-k+1+1 /(k-1)}\left(B_{r / 2}\right)$ to $u$ (evidently the positivity of $g$ is not needed for this if $k=2$ ).

It would be interesting to know whether strong convergence holds in this norm (or in some other $W^{2, q}$ norm). Of course, this question is interesting only in the range $p \leq k(n-1) / 2$. Above this range we get convergence in $C^{2, \alpha}$, by Theorem 1.2. By exploiting a term that arises in the proof of Theorem 1.1 we are able to get some information in this direction.

Theorem 1.4 Under the assumptions of Theorem 1.1 with $p>k$ we have

$$
D^{2} v_{j} \stackrel{w}{\rightarrow} D^{2} u \quad \text { in } L^{p-k+1+1 /(k-1)}\left(B_{r}\right)
$$


and

$$
\left(\Delta v_{j}\right)^{(p-k) / 2} \rightarrow(\Delta u)^{(p-k) / 2} \quad \text { in } L^{S}\left(B_{r / 2}\right)
$$

for all $s \in[1,2 n /(n-2))$.

From the viewpoint of second order elliptic equations it seems reasonable to conjecture that if $u \in W^{2, p}(\Omega)$ is a viscosity solution of (1.1), then there exist $u_{j} \in C^{\infty}\left(\bar{B}_{r}\right)$ solving approximating equations $F_{k}\left(D^{2} u_{j}\right)=g_{j}$ with $g_{j}$ satisfying the bounds in (iii), such that $u_{j} \rightarrow u$ in $W^{2, p}\left(B_{r}\right)$. Furthermore, we conjecture that this should be true with $W^{2, p}$ replaced by $C^{1, \alpha}$. On the other hand, the nonlinearity of the equations may somehow preclude this. An example of Malý [8] is interesting in this regard. He has constructed a mapping $U$ from $B_{1} \subset \mathbf{R}^{2}$ into $\mathbf{R}^{2}$ such that $\operatorname{det} D U=0$ except at 0 , $|D U(x)| \leq C|x|^{-1}$, and $U \in W^{1, p}\left(B_{1} ; \mathbf{R}^{2}\right)$ for all $p \in(1,2)$, and such that if $U_{j}$ is a sequence of Lipschitz mappings with $U_{j} \rightarrow U$ strongly in $W^{1, q}\left(B_{1} ; \mathbf{R}^{2}\right)$ for some $q \in(1, p)$, then

$$
\lim _{j \rightarrow \infty} \int_{B_{1}}\left|\operatorname{det} D U_{j}\right|^{q} \rightarrow \infty .
$$

\section{Proofs of results}

Let $x_{0}$ be any point of $\Omega$. We may assume that $x_{0}$ is the origin and that $r>0$ is so small that $B_{2 r}=B_{2 r}(0) \subset \subset \Omega$. Let $u \in W^{2, p}(\Omega)$ be a viscosity solution of (1.1). Under the regularity assumptions on $g$ it is known that $u$ belongs to $C^{0,1}(\Omega)[10,12]$. Let $u_{\epsilon}$ denote the regularization of $u$, defined by

$$
u_{\epsilon}(x)=\epsilon^{-n} \int_{\Omega} \phi\left(\frac{x-y}{\epsilon}\right) u(y) \mathrm{d} y
$$

for some nonnegative rotationally symmetric function $\phi \in C_{0}^{\infty}\left(\mathbf{R}^{n}\right)$ with support in $B_{1}(0)$ and $\int \phi=1$. Then $u_{\epsilon} \rightarrow u$ locally uniformly in $\Omega$, in $W_{\text {loc }}^{1, q}(\Omega)$ for all $q<\infty$, and in $W_{\text {loc }}^{2, p}(\Omega)$. Furthermore, for any $\Omega^{\prime} \subset \subset \Omega$ we have

$$
\sup _{\Omega^{\prime}}\left|D u_{\epsilon}\right| \leq \sup _{\Omega^{\prime}}|D u|
$$

and

$$
\int_{\Omega^{\prime}}\left|D^{2} u_{\epsilon}\right|^{p} \leq \int_{\Omega^{\prime}}\left|D^{2} u\right|^{p}
$$

provided $\epsilon<\operatorname{dist}\left(\Omega^{\prime}, \partial \Omega\right)$.

By the mean value theorem for integrals, for each sufficiently small $\epsilon>0$ there is a number $r_{\epsilon} \in[r, 2 r]$ such that

$$
\int_{\partial B_{r_{\epsilon}}}\left|D^{2} u_{\epsilon}\right|^{p} \leq \frac{1}{r_{\epsilon}} \int_{B_{2 r_{\epsilon}}}\left|D^{2} u_{\epsilon}\right|^{p} .
$$

Here integrals over $\partial B_{r_{\epsilon}}$ are always assumed to be taken with respect to the $n-1$ dimensional measure on $\partial B_{r_{\epsilon}}$; usually we will not indicate the volume elements. 
Let $\mu=\sup _{B_{2 r}}|D g|$ and define

$$
h_{\epsilon}(x)=(\mu+1) \epsilon+\epsilon^{-n} \int \phi\left(\frac{x-y}{\epsilon}\right) g(y) \mathrm{d} y .
$$

Then $h_{\epsilon} \rightarrow g$ uniformly on $B_{r}$ as $\epsilon \rightarrow 0$ and

$$
h_{\epsilon}(x) \geq(\mu+1) \epsilon+\inf _{B_{\epsilon}(x)} g \geq \epsilon+g(x)
$$

and

$$
h_{\epsilon}(x) \leq(\mu+1) \epsilon+\sup _{B_{\epsilon}(x)} g \leq(2 \mu+1) \epsilon+g(x)
$$

so

$$
\inf _{B_{r}} h_{\epsilon} \geq \inf _{B_{2 r}} g
$$

and

$$
\sup _{B_{r}}\left|D h_{\epsilon}\right| \leq \sup _{B_{2 r}}|D g|
$$

if $\epsilon<r$.

Now let $v_{\epsilon} \in C^{\infty}\left(\bar{B}_{r_{\epsilon}}\right)$ be the unique $k$-convex solution of

$$
\begin{aligned}
F_{k}\left(D^{2} v_{\epsilon}\right) & =h_{\epsilon}(x) \quad \text { in } B_{r_{\epsilon}}, \\
v_{\epsilon} & =u_{\epsilon} \quad \text { on } \partial B_{r_{\epsilon}} .
\end{aligned}
$$

Such solutions $v_{\epsilon}$ exist by the results of [1]. Furthermore, since $u_{\epsilon} \rightarrow u$ uniformly on $B_{2 r}$ and $h_{\epsilon} \rightarrow g$, we have $v_{\epsilon} \rightarrow u$ on $B_{r}$. Indeed, given any $\delta>0$, we have $\left|u_{\epsilon}-u\right|<\delta$ on $B_{2 r}$ for small enough $\epsilon$, and therefore also $\left|v_{\epsilon}-u\right|<\delta$ on $\partial B_{r_{\epsilon}}$. Then $u+\delta$ is a viscosity supersolution of (2.6) for small enough $\epsilon$ and therefore $v_{\epsilon} \leq u+\delta$ in $B_{r_{\epsilon}}$ by the comparison principle for viscosity solutions.

Next we show that $\underline{u}=u-\delta+\theta\left(|x|^{2}-r_{\epsilon}^{2}\right)$ is a viscosity subsolution of (2.6) for a suitable choice of $\theta>0$. Suppose $\underline{\psi \in C^{2}}$ is a function such that $\underline{\psi} \geq \underline{u}$ in $B_{r_{\epsilon}}$ with equality at some point $\bar{x} \in B_{r_{\epsilon}}$. Then $\psi=\psi+\delta-\theta\left(|x|^{2}-r_{\epsilon}^{2}\right)$ is a $C^{2}$ function with $\psi \geq u$ in $B_{r_{\epsilon}}$ with equality at $\bar{x}$, so at $\bar{x}$ we have $F_{l}\left(D^{2} \psi\right) \geq 0$ for all $l=0, \ldots, k$ and $F_{k}\left(D^{2} \psi\right) \geq g$. Therefore at $\bar{x}$

$$
\begin{aligned}
F_{k}\left(D^{2} \underline{\psi}\right) & =F_{k}\left(D^{2} \psi+2 \theta I\right) \geq \sum_{j=0}^{k}(2 \theta)^{j} F_{k-j}\left(D^{2} \psi\right) \\
& \geq F_{k}\left(D^{2} \psi\right)+(2 \theta)^{k} \geq g+(2 \theta)^{k} \geq h_{\epsilon}
\end{aligned}
$$

if we choose $(2 \theta)^{k}=(2 \mu+1) \epsilon$. Thus $\underline{u}$ is a viscosity subsolution of (2.6) and therefore, by the comparison principle, $\underline{u} \leq v_{\epsilon}$ in $B_{r_{\epsilon}}$. Thus

$$
v_{\epsilon}-\delta \leq u \leq v_{\epsilon}+\delta+C(k, \mu) \epsilon^{1 / k} r_{\epsilon}^{2} \leq v_{\epsilon}+2 \delta
$$

in $B_{r_{\epsilon}}$ for small enough $\epsilon>0$. It follows that $v_{\epsilon} \rightarrow u$ as $\epsilon \rightarrow 0$.

Next we derive some preliminary estimates that will be used later. First, for any constant $m>0$ and for $\underline{u}_{\epsilon}=u_{\epsilon}+m\left(|x|^{2}-r_{\epsilon}^{2}\right.$, we have

$$
F_{k}\left(D^{2} \underline{u}_{\epsilon}\right)=F_{k}\left(D^{2} u_{\epsilon}+2 m I\right) \geq(2 m)^{k},
$$


since $F_{l}\left(D^{2} u_{\epsilon}\right) \geq 0$ for $l=1, \ldots, k$. For $m$ fixed sufficiently large we have $F_{k}\left(D^{2} \underline{u}_{\epsilon}\right) \geq$ $F_{k}\left(D^{2} v_{\epsilon}\right)$ in $B_{r_{\epsilon}}$ and $\underline{u}_{\epsilon}=v_{\epsilon}$ on $\partial B_{r_{\epsilon}}$. By the comparison principle we have $\underline{u}_{\epsilon} \leq v_{\epsilon} \leq$ $\bar{u}_{\epsilon}$ in $B_{r_{\epsilon}}$, where $\bar{u}_{\epsilon}$ denotes the harmonic function in $B_{r_{\epsilon}}$ equal to $u_{\epsilon}$ on $\partial B_{r_{\epsilon}}$. Thus

$$
\sup _{B_{r_{\epsilon}}}\left|v_{\epsilon}\right| \leq C\left(n, r,\left\|h_{\epsilon}\right\|_{C^{0}\left(\bar{B}_{r}\right)}\right) .
$$

Since $\underline{u}_{\epsilon}=v_{\epsilon}$ on $\partial B_{r_{\epsilon}}$, we also have

$$
D_{v}\left(\underline{u}_{\epsilon}-v_{\epsilon}\right) \geq 0 \quad \text { on } \partial B_{r_{\epsilon}}
$$

where $v$ denotes the outer unit normal vector field to $\partial B_{r_{\epsilon}}$. Therefore

$$
\begin{aligned}
\sup _{\partial B_{r_{\epsilon}}} D_{\nu} v_{\epsilon} & \leq \sup _{\partial B_{r_{\epsilon}}} D_{\nu} \underline{u}_{\epsilon} \\
& \leq \sup _{B_{2 r}}\left|D u_{\epsilon}\right|+4 m r \\
& \leq C\left(k, n, r,\left\|h_{\epsilon}\right\|_{C^{0}\left(\bar{B}_{2 r}\right)},\left\|D u_{\epsilon}\right\|_{C^{0}\left(\bar{B}_{2 r}\right)}\right) .
\end{aligned}
$$

Next, the interior gradient estimate of $[2,11]$ implies that

$$
\sup _{B_{r_{\epsilon} / 2}}\left|D v_{\epsilon}\right| \leq C\left(n, r,\left\|h_{\epsilon}\right\|_{C^{1}\left(\bar{B}_{r}\right)}\right) \text {. }
$$

Our goal now is to show that

$$
\int_{B_{r_{\epsilon} / 2}}\left(\Delta v_{\epsilon}\right)^{p-k+1} F_{k-1}\left(D^{2} v_{\epsilon}\right)
$$

is also bounded independently of $\epsilon$. We will achieve this by combining some ideas from [15] with an observation of Trudinger [10].

We temporarily drop the subscripts $\epsilon$ on $v, h$ and $r$ to simplify notation. On the ball $B_{r}$ we consider the function

$$
w=\left(\delta_{k l}-\frac{x_{k} x_{l}}{r^{2}}\right) D_{k l} v .
$$

By direct computation using the concavity of $F_{k}\left(D^{2} v\right)^{1 / k}$ (which can be written as $\left.-F_{i j, r s} \geq\left(\frac{1}{k}-1\right) F^{-1} F_{i j} F_{r s}\right)$, we find, as in [10],

$$
\begin{aligned}
F_{i j} D_{i j} w \geq & \left(\delta_{k l}-\frac{x_{k} x_{l}}{r^{2}}\right) D_{k l} h \\
& +\left(k^{-1}-1\right)\left(\delta_{k l}-\frac{x_{k} x_{l}}{r^{2}}\right) \frac{D_{k} h D_{l} h}{h}-\frac{4 D_{k} h x_{k}}{r^{2}}-\frac{2 k h}{r^{2}} .
\end{aligned}
$$

Therefore

$$
F_{i j} D_{i j} w \geq\left(\delta_{k l}-\frac{x_{k} x_{l}}{r^{2}}\right) D_{k l} h-C(k, n) \mathscr{Z}
$$

where

$$
\mathscr{Z}:=\frac{|D h|^{2}}{h}+\frac{|D h|}{r}+\frac{h}{r^{2}} .
$$


We now let $q \geq 0$ and multiply (2.11) by $\eta w^{q}$ where $\eta=\left(r^{2}-|x|^{2}\right)$. Then we have

$$
\int_{B_{r}} \eta w^{q} F_{i j} D_{i j} w \geq \int_{B_{r}} \eta w^{q}\left(\delta_{k l}-\frac{x_{k} x_{l}}{r^{2}}\right) D_{k l} h-C \int_{B_{r}} \eta w^{q} \mathscr{Z} .
$$

Integrating the left hand side by parts twice and using the divergence structure of $F_{i j}$, $D_{i} F_{i j}=0$, we have

$$
\begin{aligned}
& -C \int_{B_{r}} \eta w^{q} \mathscr{Z}+\int_{B_{r}} \eta w^{q}\left(\delta_{k l}-\frac{x_{k} x_{l}}{r^{2}}\right) D_{k l} h \\
& \leq-q \int_{B_{r}} \eta w^{q-1} F_{i j} D_{i} w D_{j} w-\frac{1}{q+1} \int_{B_{r}} F_{i j} D_{i} \eta D_{j}\left(w^{q+1}\right) \\
& =-q \int_{B_{r}} \eta w^{q-1} F_{i j} D_{i} w D_{j} w+\frac{1}{q+1} \int_{B_{r}} w^{q+1} F_{i j} D_{i j} \eta \\
& \quad-\frac{1}{q+1} \int_{\partial B_{r}} w^{q+1} F_{i j} D_{i} \eta v_{j}
\end{aligned}
$$

where $v=x / r$ is the outer unit normal to $\partial B_{r}$. Using the facts that $D_{i} \eta=-2 x_{i}$, $D_{i j} \eta=-2 \delta_{i j}$ and

$$
\sum F_{i i}=(n-k+1) F_{k-1}
$$

we arrive at the estimate

$$
\begin{aligned}
& \frac{1}{q+1} \int_{B_{r}} w^{q+1} F_{k-1}\left(D^{2} v\right)+\frac{q}{2(n-k+1)} \int_{B_{r}} \eta w^{q-1} F_{i j}\left(D^{2} v\right) D_{i} w D_{j} w \\
& \leq \frac{r}{(q+1)(n-k+1)} \int_{\partial B_{r}} w^{q+1} F_{v v}\left(D^{2} v\right)+C \int_{B_{r}} \eta w^{q} \mathscr{Z} \\
& \quad+\frac{1}{2(n-k+1)} \int_{B_{r}} \eta w^{q}\left(\delta_{k l}-\frac{x_{k} x_{l}}{r^{2}}\right) D_{k l} h .
\end{aligned}
$$

Next we handle the last term on the right. Integrating by parts we get

$$
\begin{aligned}
& \int_{B_{r}} \eta w^{q}\left(\delta_{k l}-\frac{x_{k} x_{l}}{r^{2}}\right) D_{k l} h \\
& \quad=-\int_{B_{r}}\left(D_{k} \eta w^{q}+\eta q w^{q-1} D_{k} w\right)\left(\delta_{k l}-\frac{x_{k} x_{l}}{r^{2}}\right) D_{l} h+\int_{B_{r}} \eta w^{q} D_{k}\left(\frac{x_{k} x_{l}}{r^{2}}\right) D_{l} h .
\end{aligned}
$$

All the terms except the one containing $D w$ can be estimated in a straightforward way: they are controlled by

$$
C(k, n) \int_{B_{r}} r w^{q}|D h| \leq C(k, n) r^{2} \int_{B_{r}} w^{q} \mathscr{Z} .
$$


Next, writing $D_{k}^{T} h=\left(\delta_{k l}-\frac{x_{k} x_{l}}{r^{2}}\right) D_{l} h$, we estimate

$$
\begin{aligned}
& q\left|\int_{B_{r}} \eta w^{q-1} D_{k}^{T} h D_{k} w\right| \\
& \quad \leq \frac{q}{4(n-k+1)} \int_{B_{r}} \eta w^{q-1} F_{i j} D_{i} w D_{j} w+C(k, n) q \int_{B_{r}} \eta w^{q-1} F^{i j} D_{i}^{T} h D_{j}^{T} h \\
& \quad \leq \frac{q}{4(n-k+1)} \int_{B_{r}} \eta w^{q-1} F_{i j} D_{i} w D_{j} w+C(k, n) q \int_{B_{r}} \eta \frac{|D h|^{2}}{h} w^{q-1} \Delta v
\end{aligned}
$$

where $\left[F^{i j}\right]$ denotes the inverse matrix of $\left[F_{i j}\right]$, and we have used the estimate

$$
\left[F^{i j}\right] \leq \frac{\Delta v}{h} I
$$

(see $[14,15]$ ). The first term on the right can be absorbed by the second term on the left side of (2.13). Next, writing $\hat{x}=x /|x|$ for $x \neq 0$, we have

$$
w=\Delta v-\frac{|x|^{2}}{r^{2}} D_{\hat{x} \hat{x}} v \geq\left(1-\frac{|x|^{2}}{r^{2}}\right) \Delta v=r^{-2} \eta \Delta v,
$$

and this is clearly also true at $x=0$. Here we have used the fact that if $\lambda_{1} \geq \cdots \geq \lambda_{n}$ are the eigenvalues of $D^{2} v$, then $D_{\hat{x} \hat{x}} v \leq \lambda_{1}$ and therefore

$$
\Delta v-D_{\hat{x} \hat{x}} v \geq \sum_{j=2}^{n} \lambda_{j}>0
$$

since $\lambda \in \Gamma_{2}$. We deduce therefore that

$$
\int_{B_{r}} \eta w^{q-1} \Delta v \frac{|D h|^{2}}{h} \leq r^{2} \int_{B_{r}} w^{q} \frac{|D h|^{2}}{h} .
$$

Combining the above estimates we have, with $\epsilon$ now reinstated,

$$
\begin{aligned}
& \frac{1}{q+1} \int_{B_{r_{\epsilon}}} w_{\epsilon}^{q+1} F_{k-1}\left(D^{2} v_{\epsilon}\right)+\frac{q}{4(n-k+1)} \int_{B_{r_{\epsilon}}} \eta w_{\epsilon}^{q-1} F_{i j}\left(D^{2} v_{\epsilon}\right) D_{i} w_{\epsilon} D_{j} w_{\epsilon} \\
& \leq \frac{r_{\epsilon}}{(q+1)(n-k+1)} \int_{\partial B_{r_{\epsilon}}} w_{\epsilon}^{q+1} F_{\nu v}\left(D^{2} v_{\epsilon}\right)+C r_{\epsilon}^{2} \int_{B_{r_{\epsilon}}} w_{\epsilon}^{q} \mathscr{Z}_{\epsilon},
\end{aligned}
$$

where $C$ depends only on $k, n$ and $q$, and

$$
\mathscr{Z}_{\epsilon}:=\frac{\left|D h_{\epsilon}\right|^{2}}{h_{\epsilon}}+\frac{\left|D h_{\epsilon}\right|}{r_{\epsilon}}+\frac{h_{\epsilon}}{r_{\epsilon}^{2}} .
$$

Next we estimate the boundary integral in (2.14). We choose any point $\xi \in \partial B_{r_{\epsilon}}$ and rotate coordinates so that $\xi=\left(0, \ldots, 0, r_{\epsilon}\right)$. Since $v_{\epsilon}=\underline{u}_{\epsilon}$ on $\partial B_{r_{\epsilon}}$, at $\xi$ we have, for $\alpha, \beta=1, \ldots, n-1$,

$$
D_{\alpha \beta}\left(v_{\epsilon}-\underline{u}_{\epsilon}\right)=D_{n}\left(v_{\epsilon}-\underline{u}_{\epsilon}\right) \frac{\delta_{\alpha \beta}}{r_{\epsilon}},
$$


and therefore, by (2.8),

$$
\left[D_{\alpha \beta} v_{\epsilon}\right] \leq\left[D_{\alpha \beta} \underline{u}_{\epsilon}\right] \quad \text { at } \quad \xi
$$

in the sense of matrices. Since $\lambda\left(D^{2} v_{\epsilon}\right) \in \Gamma_{k} \subset \Gamma_{k-1} \subset \cdots \subset \Gamma_{1}$, we have, at $\xi$,

$$
F_{l-1}\left(\widehat{D^{2} v_{\epsilon}}\right)=\frac{\partial}{\partial\left(D_{\nu v} v_{\epsilon}\right)} F_{l}\left(D^{2} v_{\epsilon}\right)>0 \quad \text { for all } l=1, \ldots, k
$$

where $\widehat{D^{2} v_{\epsilon}}$ denotes $\left[D_{\alpha \beta} v_{\epsilon}\right]_{\alpha, \beta<n}$. It follows from this that $\lambda\left(\widehat{D^{2} v_{\epsilon}}\right) \in \Gamma_{k-1}^{n-1}$, the $k-1$ admissible cone in $\mathbf{R}^{n-1}$. Similarly, $\lambda\left(\widehat{D^{2} \underline{u}_{\epsilon}}\right) \in \Gamma_{k-1}^{n-1}$. Therefore (2.15) implies that

$$
F_{\nu v}\left(D^{2} v_{\epsilon}\right) \leq F_{\nu v}\left(D^{2} \underline{u}_{\epsilon}\right) \text { at } \xi \text {. }
$$

Since $\xi \in \partial B_{r_{\epsilon}}$ is arbitrary, it follows that

$$
\begin{aligned}
F_{\nu \nu}\left(D^{2} v_{\epsilon}\right) & \leq C(k, n)\left(\Delta^{\prime} \underline{u}_{\epsilon}\right)^{k-1} \\
& \leq \widehat{C}(k, n)\left(1+\left(\Delta^{\prime} u_{\epsilon}\right)^{k-1}\right) \quad \text { on } \quad \partial B_{r_{\epsilon}} .
\end{aligned}
$$

where $\Delta^{\prime}=\Delta-D_{\nu v}$ on $\partial B_{r_{\epsilon}}$. Therefore by (2.5),

$$
\begin{aligned}
r_{\epsilon} \int_{\partial B_{r_{\epsilon}}} w_{\epsilon}^{q+1} F_{\nu \nu}\left(D^{2} v_{\epsilon}\right) & \leq C(k, n, q) r_{\epsilon} \int_{\partial B_{r_{\epsilon}}}\left(1+\left(\Delta^{\prime} u_{\epsilon}\right)^{q+k}\right) \\
& \leq C(k, n, q) \int_{B_{2 r_{\epsilon}}}\left(1+\left|D^{2} u_{\epsilon}\right|^{q+k}\right)
\end{aligned}
$$

if $q+k=p$.

Using this in (2.14) we obtain

$$
\begin{gathered}
\int_{B_{r_{\epsilon}}} w_{\epsilon}^{q+1} F_{k-1}\left(D^{2} v_{\epsilon}\right)+\int_{B_{r_{\epsilon}}} \eta w_{\epsilon}^{q-1} F_{i j}\left(D^{2} v_{\epsilon}\right) D_{i} w_{\epsilon} D_{j} w_{\epsilon} \\
\leq C \int_{B_{2 r_{\epsilon}}}\left(1+\left|D^{2} u_{\epsilon}\right|^{q+k}\right)+C r_{\epsilon}^{2} \int_{B_{r_{\epsilon}}} w_{\epsilon}^{q} \mathcal{Z}_{\epsilon},
\end{gathered}
$$

where $C$ depends only on $k, n$ and $q$. This is the key estimate from which our results follow.

To derive Theorem 1.1 we discard the second term on the left and we estimate

$$
\mathcal{Z}_{\epsilon} \leq \frac{2}{r_{\epsilon}^{2}}\left(\sup _{B_{r_{\epsilon}}} \frac{\left|D h_{\epsilon}\right|^{2}}{h_{\epsilon}}+\sup _{B_{r_{\epsilon}}} h_{\epsilon}\right),
$$

which in turn can be controlled by the corresponding quantity with $h_{\epsilon}$ replaced by $g$. Using (1.2) (with $g$ replaced by $h_{\epsilon}$ ) and the fact that $h_{\epsilon}$ has a positive lower bound if $g$ is positive, we can then absorb the term $\int_{B_{r_{\epsilon}}} w_{\epsilon}^{q}$ into the left hand side of (2.16) at the expense of leaving a large constant on the right, to obtain

$$
\int_{B_{r_{\epsilon}}} w_{\epsilon}^{q+1} F_{k-1}\left(D^{2} v_{\epsilon}\right) \leq C\left(1+\int_{B_{r_{\epsilon}}}\left|D^{2} u_{\epsilon}\right|^{q+k}\right) .
$$


where $C$ depends on $k, n, q, r$ and on $\|g\|_{C^{0,1}\left(\bar{B}_{r_{\epsilon}}\right)}$ and $\inf _{B_{r_{\epsilon}}} g>0$. Next, on $B_{r_{\epsilon} / 2}$ we have

$$
w_{\epsilon} \geq \frac{3}{4} \Delta v_{\epsilon}
$$

so we conclude, after using (2.4), that

$$
\int_{B_{r / 2}}\left(\Delta v_{\epsilon}\right)^{p-k+1} F_{k-1}\left(D^{2} v_{\epsilon}\right) \leq C\left(1+\int_{B_{r}}\left|D^{2} u\right|^{p}\right) .
$$

Remark 2.1 If $q=0$ (i.e., $p=k$ ), there is no need to absorb the term $\int_{B_{r_{\epsilon}}} w_{\epsilon}^{q}$ into the left side of (2.16), while if $k=2$, then $F_{k-1}\left(D^{2} v_{\epsilon}\right)=\Delta v_{\epsilon} \geq w_{\epsilon}$. In each of these cases the constant $C$ in (2.16) does not depend on a positive lower bound for $g$; in fact $C$ depends only on $k, n, q$ and $\left\|\tilde{h}_{\epsilon}\right\|_{C^{0,1}\left(\bar{B}_{r_{\epsilon}}\right)}$ where $\tilde{h}_{\epsilon}=h_{\epsilon}^{1 / 2}$.

To prove Theorem 1.3 we first modify the proof of Theorem 1.1 slightly. Recalling our assumption $\tilde{g}=\left(\operatorname{det} D^{2} u\right)^{1 / 2} \in W^{1, p}(\Omega), p \geq 2$, we define

$$
\tilde{h}_{\epsilon}(x)=\epsilon+\epsilon^{-n} \int \phi\left(\frac{x-y}{\epsilon}\right) \tilde{g}(y) d y .
$$

Then $\tilde{h}_{\epsilon}$ is bounded in $L^{p}\left(\Omega^{\prime}\right)$ for any $\Omega^{\prime} \subset \subset \Omega$ and $\epsilon<\operatorname{dist}\left(\Omega^{\prime} \partial \Omega\right.$ ) (this is not quite evident for the approximations used above), and $h_{\epsilon}:=\tilde{h}_{\epsilon}^{2} \rightarrow g:=\tilde{g}^{2}$ in $L_{l o c}^{1}(\Omega)$. It follows from standard results on Monge-Ampère equations that if $v_{\epsilon}$ is defined by (2.6) as before, then $v_{\epsilon} \rightarrow u$ locally uniformly on $B_{r_{\epsilon}}$ (we do not need to use the argument from above). Furthermore, the convexity of $v_{\epsilon}$ implies that $\sup _{B_{r_{\epsilon} / 2}}\left|D v_{\epsilon}\right|$ is bounded independently of $\epsilon$. We now follow the proof of Theorem 1.1 and estimate the last term in (2.16) in an obvious way using the Hölder and Young inequalities and the fact that $\mathscr{Z}_{\epsilon}$ is bounded in $L^{p / 2}\left(B_{r_{\epsilon}}\right)$ :

$$
\int_{B_{R_{\epsilon}}} w_{\epsilon}^{q} \mathscr{Z}_{\epsilon}=\int_{B_{R_{\epsilon}}} w_{\epsilon}^{p-2} \mathscr{Z}_{\epsilon} \leq \delta \int_{B_{R_{\epsilon}}} w_{\epsilon}^{p}+C(\delta) \int_{B_{R_{\epsilon}}} \mathscr{Z}_{\epsilon}^{p / 2} .
$$

Theorem 1.3 then follows by applying the estimate in [16] to the approximations $v_{\epsilon}$.

Remark 2.2 We have not been able to extend the estimate in [16] for smooth solutions to $W^{2, p}$ solutions if $p \in(1,2)$. The main difficulty in this case is that the second term on the left in (2.16) has a negative coefficient (depending on $p$ ) and it needs to be controlled by the concavity term coming from the twice differentiated equation. More precisely, we would need to show that

$$
\begin{aligned}
& -w_{\epsilon} F_{i j, r s} D_{i j k} v_{\epsilon} D_{r s l} v_{\epsilon}\left(\delta_{k l}-\frac{x_{k} x_{l}}{r^{2}}\right) \\
& \quad \geq(1-\delta) F_{i j}\left(\delta_{k l}-\frac{x_{k} x_{l}}{r^{2}}\right) D_{i k l} v_{\epsilon}\left(\delta_{p q}-\frac{x_{p} x_{q}}{r^{2}}\right) D_{j p q} v_{\epsilon}
\end{aligned}
$$

for some $\delta \in(0,1)$. This is true at $x=0$, as shown in [16], but we have not been able to prove it in general. 
Finally, we show how Theorem 1.4 follows. The first part of the theorem is evident from Theorem 1.1 and the estimate (1.2). To prove the second part we use the second term on the left side of (2.16). Using the fact that $h_{\epsilon}$ has a positive lower bound, together with the facts that

$$
\left[F_{i j}\left(D^{2} v_{\epsilon}\right)\right] \geq \frac{h_{\epsilon}}{\Delta v_{\epsilon}} I
$$

and

$$
\Delta v_{\epsilon} \geq w_{\epsilon} \geq r_{\epsilon}^{-2} \eta \Delta v_{\epsilon},
$$

which have already been used above, we compute

$$
\begin{aligned}
& \int_{B_{r_{\epsilon}}} \eta w_{\epsilon}^{q} F_{i j}\left(D^{2} v_{\epsilon}\right) D_{i} w_{\epsilon} D_{j} w_{\epsilon} \\
& \geq c_{0} \int_{B_{r_{\epsilon} / 2}} w_{\epsilon}^{q-2}\left|D w_{\epsilon}\right|^{2} \\
& =\frac{4 c_{0}}{q^{2}} \int_{B_{r_{\epsilon} / 2}}\left|D\left(w_{\epsilon}^{q / 2}\right)\right|^{2} \\
& \geq \frac{c_{1}}{q^{2}} \int_{B_{r_{\epsilon} / 4}}\left|D\left(\left(\Delta v_{\epsilon}\right)^{q / 2}\right)\right|^{2}-C \int_{B_{r_{\epsilon}}} w_{\epsilon}^{q}
\end{aligned}
$$

for controlled positive constants $c_{0}, c_{1}, C$. Using this in (2.16) we see that

$$
\left.\int_{B_{r_{\epsilon} / 4}} \mid D\left(\left(\Delta v_{\epsilon}\right)^{q / 2}\right)\right)\left.\right|^{2}+\int_{B_{r_{\epsilon} / 4}}\left(\Delta v_{\epsilon}\right)^{q+1+1 /(k-1)}
$$

is bounded independently of $\epsilon$. By the Rellich-Kondrachov compactness theorem [5, Theorem 7.26], we may therefore assume that for a suitable subsequence $\left\{\epsilon_{j}\right\}$ decreasing to zero, $\left(\Delta v_{\epsilon_{j}}\right)^{q / 2}$ converges strongly in $L^{s}\left(B_{r / 4}\right)$ for all $s \in[1,2 n /(n-2))$. Standard arguments using the first part of Theorem 1.4 then imply that the limit is $(\Delta u)^{q / 2}$, so the second assertion of Theorem 1.4 is proved.

\section{References}

1. Caffarelli, L., Nirenberg, L., Spruck, J.: The Dirichlet problem for nonlinear second-order elliptic equations. III. Functions of the eigenvalues of the Hessian. Acta Math. 155, 261-301 (1985)

2. Chou, K.-S., Wang, X.-J.: A variational theory of the Hessian equation. Comm. Pure Appl. Math. 54, 1029-1064 (2001)

3. Delanoë, P.: Hessian equations on compact non-negatively curved Riemannian manifolds. Calc. Var. Partial Differ. Equations 16, 165-176 (2003)

4. Evans, L.C.: Classical solutions of fully nonlinear, convex, second-order elliptic equations. Comm. Pure Appl. Math. 35, 333-363 (1982)

5. Gilbarg, D., Trudinger, N.S.: Elliptic partial differential equations of second order, 2nd ed, Springer Berlin (1983)

6. Krylov, N.V.: Boundedly inhomogeneous elliptic and parabolic equations. Izv. Akad. Nauk SSSR Ser. Mat. 46, 87-523, 670 (Russian) (1982) 
7. Liu, S., Bao, J.: The local regularity for strong solutions of the Hessian quotient equation. J. Math. Anal. Appl., 303, 462-476 (2005)

8. Malý, J.: $L^{p}$ approximation of Jacobians Comment. Math. Univ. Carolinae 32, 659-666 (1991)

9. Pogorelov, A.V.: The Minkowski multidimensional problem. Wiley, New York-1TorontoLondon (1978)

10. Trudinger, N.S.: On degenerate fully nonlinear elliptic equations in balls. Bull. Austral. Math. Soc. 35, 299-307 (1987)

11. Trudinger, N.S.: Weak solutions of Hessian equations. Comm. Partial Differ. Equations, 22, 1251$1261(1997)$

12. Trudinger, N.S., Wang, X.-J.: Hessian measures II. Ann. Math., 150, 579-604 (1999)

13. Urbas, J.: On the existence of nonclassical solutions for two classes of fully nonlinear elliptic equations: Indiana Univ. Math. J. 39, 355-382 (1990)

14. Urbas, J.: Some interior regularity results for solutions of Hessian equations. Calc. Var. Partial Differ. Equations 11, 1-31 (2000)

15. Urbas, J.: An interior second derivative bound for solutions of Hessian equations. Calc. Var. Partial Differ. Equations 12, 417-431 (2001)

16. Urbas, J.: Some new estimates for solutions of degenerate two dimensional Monge-Ampère equations. Math. Z. 236, 625-641 (2001) 